\title{
The Universal Coefticient Theorem in the Category of Fuzzy Soft Modules
}

\author{
S. A. Bayramov, S. E. Abdullayev
}

Department of Algebra and Geometry, Baku State University, 23, Z. Khalilov str., AZ1148, Baku, Azerbaijan

\begin{abstract}
This paper begins with the basic concepts of chain comlexes of fuzzy soft modules. Later, we introduce short exact sequence of fuzzy soft modules and prove that split short exact sequence of fuzzy soft chain complex. Naturally, we want to investigate whether or not the universal coefficient theorems are satisfied in category of fuzzy soft chain complexes. However, in the proof of these theorems in the category of chain complexes, exact sequence of homology modules of chain complexes is used. Generally, sequence of fuzzy soft homology modules is not exact in fuzzy chain complexes. Therefore in this study, we construct exact sequence of fuzzy soft homology modules under some conditions. Universal coefficients theorem is proven by making use of this idea.
\end{abstract}

Keywords: Universal Coefticient Theorem, Soft Module, Fuzzy Soft Module, Chain Complex Of Fuzzy Soft Modules, Short Exact Sequence Fuzzy Soft Modules.

Date of Publication: 2018-09-15

DOI: https://doi.org/10.24297/jam.v14i2.7599

ISSN: 2347-1921

Volume: 14 Issue: 02

Journal: Journal of Advances in Mathematics

Website: https://cirworld.com

This work is licensed under a Creative Commons Attribution 4.0 International License. 


\section{Introduction}

The concept of fuzzy sets was introduced by Lotfi A. Zadeh in 1965 [22]. Since then tht fuzzysets and fuzzy logic have been applied in many real life problems in uncertain, ambiguous environment. The idea of extending the concepts of fuzzy sets to algebra dates back to the introduction in 1971 by Rosenfeld of fuzzy subgroups of a group [11]. Later several researchers have studied fuzzy modules and then Lopez-Permouth and Malik introduced the category of $R-f z$ modof fuzzy left $R-$ modules over a ring $R$ [21]. Ameri and Zahedi defined the concept of fuzzy exact sequence in the category of fuzzy modules, and obtained some results related to these notions [15]. Same researchers have previously introduced the category of fuzzy chain complexes and determined fuzzy homology functor in the category. It was proved that this functor is invariant with respect to fuzzy homotopy given in [14]. Molodtsov [10] initiated the concept of soft set theory as a new mathematical tool for dealing with uncertainties. Later, work on the soft set theory is progressing rapidly. Maji et al. [8,9] have published a detailed theoretical study on soft sets. After Molodtsov's work, some different applications of soft sets were studied in [9]. H. Aktaş and N. Cagman [2] has established a connection betwen soft sets and fuzzy sets and they introduced soft groups. At the same time, they gave a definition of soft groups, soft rings and derived their basic properties ([1,4,7]). Qiu-Mei Sun et al. [20] defined soft modules and investigated their basic properties.

L. Jin-Liang [7] presented fuzzy soft sets and fuzzy soft groups. C. Gunduz and S. Bayramov [5] presented fuzzy soft and intuitionistic fuzzy soft modules. Universal coefficient theorem in the category of fuzzy and intuitionistic fuzzy modules proved in $[13,19]$. Naturally, we want to investigate whether or not the universal coefficient theorems are satisfied in category of fuzzy chain complexes. However, in the proof of these theorems in the category of chain complexes, exact sequence of homology modules of chain complexes is used. Generally, sequence of fuzzy homology modules is not exact in fuzzy chain complexes. Therefore, in this study, we construct exact sequence of fuzzy homology modules under some conditions. Universal coefficients theorem is proven by making use of this idea.

\section{Preminilaries}

In this section, we recall necessary information commonly used in intuitionistic fuzzy soft module.

Definition 2.1. ([17]). Let $X$ be an initial universe set and $E$ be a set of parameters. A pair $(F, E)$ is called a soft set over $X$ if only if $F$ is a mapping from $E$ into the set of all subsets of the set, $\mathrm{X}$ i.e., $F: E \rightarrow P(X)$, where $P(X)$ is the power set of $X$.

In other words, the soft set is a parameterized family of subsets of the set X. Every set $\mathrm{F}(\mathrm{e})$, for every $e \in E$, may be considered as the set of $e$-elements of the soft set $(F, E)$, or as the set of $e$-approximate elements of the soft set.

According to this manner, a soft set $(F, E)$ is given as consisting of collection of approximations:

$$
(F, E)=\{F(e): e \in E\}
$$

Definition $2.2([4,11])$. Let $I^{X}$ denote the set of all fuzzy sets on $X$ and $A \subset E$. A pair $(f, A)$ is called a fuzzy soft set over $X$, where $f$ is a mapping from $A$ into $I^{X}$. That is, for each $a \in A, f(a)=f_{a}: X \rightarrow I$, is a fuzzy set on $X$.

Definition 2.3 ([4, 11]). Union of two fuzzy soft sets $(f, A)$ and $(g, B)$ over a common universe $X$ is the fuzzy soft set $(h, C)$, where $C=A \cup B$ and 


$$
h(c)=\left\{\begin{array}{l}
f(c), \text { if } c \in A-B \\
g(c), \text { if } c \in B-A \\
f(c) \vee g(c), \text { if } c \in A \cap B
\end{array}, \quad \forall c \in C\right.
$$

It is denoted as $(f, A) \bigcup(g, B)=(h, C)$.

Definition $2.4([4,11])$. Intersection of two fuzzy soft sets $(f, A)$ and $(g, B)$ over a common universe $X$ is the fuzzy soft set $(h, C)$, where $C=A \cap B$ and $h(c)=f(c) \wedge g(c), \forall c \in C$.

It is written as $(f, A) \cap(g, B)=(h, C)$.

Definition 2.5 ([4, 11]). If $(f, A)$ and $(g, B)$ are two soft sets, then $(f, A)$ and $(g, B)$ is denoted as $(f, A) \wedge(g, B) .(f, A) \wedge(g, B)$ is defined as $(h, A \times B)$ where $h(a, b)=f(a) \wedge g(b), \forall(a, b) \in A \times B$

Now, let $M$ be a left $R$-module, $A$ be any nonemply set. $F: A \rightarrow P(M)$ refer to a set-valued function and the pair $(F, A)$ is a soft set over $M$.

Definition 2.6 ([20]). Let $(F, A)$ be a soft set over $M .(F, A)$ is said to be a soft module over $M$ if and only if $F(x)<M$ for all $x \in A$.

Definition 2.7 ([20]). Let $(F, A)$ and $(G, B)$ be two soft modules over $M$ and $N$ respectively. Then $(F, A) \times(G, B)=(H, A \times B)$ is defined as $H(x, y)=F(x) \times G(y)$ for all $(x, y) \in A \times B$.

Proposition 2.8 ([20]). Let $(F, A)$ and $(G, B)$ be two soft modules over $M$ and $N$ respectively. Then $(F, A) \times(G, B)$ is soft module over $M \times N$.

Definition 2.9 ([20]). Let $(F, A)$ and $(G, B)$ be two soft modules over $M$ and $N$ respectively, $f: M \rightarrow N, g: A \rightarrow B$ be two functions. Then we say that $(f, g)$ is a soft homomorphism if the following conditions are satisfied:

(1) $f$ is a homomorphism from $M$ onto $N$,

(2) $g$ is a mapping from $A$ onto $B$, and

(3) $f(F(x))=G(g(x))$ for all $x \in A$.

Definition 2.10 ([11]). Let $(F, A)$ be a fuzzy soft set over $M$. Then $(F, A)$ is said to be a fuzzy soft module over $M$ iff for each $a \in A, F(a)$ is a fuzzy submodule of $M$

Definition 2.11. Let $\mu_{A}$ be a right fuzzy $\Lambda$ - module and let $v_{B}$ be a left fuzzy $\Lambda$-module. Given a fuzzy projective presentation

$$
\overline{0} \rightarrow \mu_{0_{\mathrm{R}}} \stackrel{\overline{\mathrm{f}}}{\rightarrow} \mu_{0_{\mathrm{r}}} \stackrel{\overline{\mathrm{g}}}{\rightarrow} \mu_{\mathrm{A}} \rightarrow \overline{0}
$$

Of $\mu_{A}$, we define 


$$
\mathrm{F}-\operatorname{Tor}_{\overline{\mathrm{g}}}^{\wedge} \quad\left(\mu_{\mathrm{A}}, \mathrm{v}_{\mathrm{B}}\right)=\operatorname{ker}\left(\tilde{\mathrm{f}}=\tilde{\mathrm{f}} \otimes \tilde{1}:\left(\mu_{0} \otimes v\right)_{\mathrm{R} \otimes_{\Lambda}} \mathrm{B} \rightarrow\left(\mu_{0} \otimes v\right)_{\mathrm{r} \otimes_{\Lambda}} \mathrm{B}\right) .
$$

Thus, the fuzzy sequence

$\left.\left.\overline{0} \rightarrow \mathrm{F}-\operatorname{Tor}_{\overline{\mathrm{g}}} \wedge \quad\left(\mu_{\mathrm{A}}, \mathrm{v}_{\mathrm{B}}\right) \stackrel{\overline{\mathrm{I}}}{\rightarrow}\left(\mu_{0} \otimes \mathrm{v}\right)_{\mathrm{R} \otimes_{\Lambda}} \mathrm{B} \stackrel{\overline{\mathrm{f}} \otimes \overline{\mathrm{I}}}{\longrightarrow}\left(\mu_{0} \otimes \mathrm{v}\right)_{\mathrm{P} \otimes_{\wedge \mathrm{B}}}\right) \stackrel{\overline{\mathrm{g}} \otimes \overline{\mathrm{I}}}{\longrightarrow}(\mu \otimes \mathrm{v})_{\mathrm{A} \otimes \wedge \mathrm{B}}\right) \rightarrow \overline{0}$.

is exact.

\section{Chain complexes of fuzzy soft modules.}

Let be for $\forall n \in Z\left(F_{n}, A\right)$ - is fuzzy soft module over module $M_{n}$ and $\left(\partial_{n}, 1_{A}\right):\left(F_{n}, A\right) \rightarrow\left(F_{n-1}, A\right)$ is homomorphism of fuzzy soft modules.

Definition 3.1. If for all $a \in A$

$$
\left\{\left(M_{n}, F_{n}(a), \partial_{n}:\left(M_{n}, F_{n}(a)\right) \rightarrow\left(M_{n-1}, F_{n-1}(a)\right\}\right.\right.
$$

is a chain complexes of fuzzy modules, then the following sequence is said to be a chain complex of fuzzy soft modules

$$
\left\{\left(F_{n}, A\right),\left(\partial_{n},\left(I_{A}\right):\left(F_{n}, A\right) \rightarrow\left(F_{n-1} A\right)\right\}\right.
$$

Definition 3.2. If the condition $J_{m} \partial_{n}=k e r \partial_{n-1}$ is satisfied at the chain complex $\left\{\left(M_{n}, F_{n}(a), \partial_{n}:\left(M_{n}, F_{n}(a)\right) \rightarrow\left(M_{n-1}, F_{n-1}(a)\right\}\right.\right.$, then the sequence $(1)$ is said to be an exact sequence of fuzzy soft modules.

Now, let us define morphisms of the chain complexes of fuzzy soft modules.

Definition 3.3. Let $\left\{\left(F_{n}, A\right), \partial_{n}\right\},\left\{\left(G_{n}, B\right), \partial_{n}^{\prime}\right\}$ be chain complexes of soft modules over $\left\{M_{n}\right\}$ and $\left\{N_{n}\right\}$, respectively, $\left\{f_{n}: M_{n} \rightarrow N_{n}\right\}_{n}$ be homomorphism of modules and $g: A \rightarrow B$ is a mapping of sets. If the following diagram is commutative, for each $a \in A$

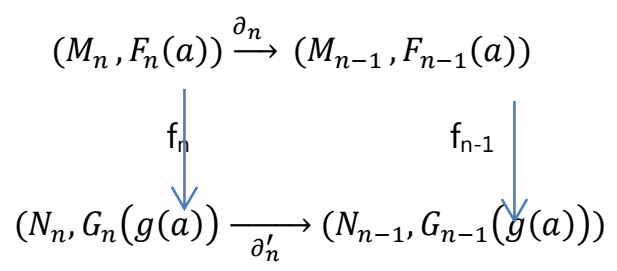

then $\left(\left\{f_{n}\right\}, g\right):\left\{\left(F_{n}, A\right), \partial_{n}\right\} \rightarrow\left\{\left(G_{n}, B\right), \partial_{n}^{\prime}\right\}$ is said to be morphism of chain complexes of fuzzy soft modules.

Chain complexes of fuzzy soft modules and morphisms of their forms a category. This category is denoted by CCSM.

Definition 3.4. Let $\left(\left\{\varphi_{n}\right\}, g\right):\left(\left\{\psi_{n}\right\}, g\right):\left\{\left(F_{n}, A\right), \partial_{n}\right\} \rightarrow\left\{\left(G_{n}, B\right), \partial_{n}^{\prime}\right\}$ be morphisms of chain complex of fuzzy soft modules and let $D=\left\{\left(D_{n}, g\right):\left(F_{n}, A\right) \rightarrow\left(G_{n+1}, B\right)\right\}$ be a family of homomorphisms of fuzzy soft modules. If the equation $\varphi_{n}-\psi_{n}=D_{n-1} \circ \partial_{n}+\partial_{n+1}^{\prime} \circ D_{n}$ is satisfied, then the family of homomorphisms of modules $D=\left\{\left(D_{n}, g\right): M_{n} \rightarrow N_{n+1}\right\}_{n \in Z}$ is said to be a chain homotopy morphisms, $\left(\left\{\varphi_{n}\right\}, g\right),\left(\left\{\psi_{n}\right\}, g\right)$ is said to be a chain homotopy morphisms and denoted by $\left(\left\{\varphi_{n}\right\}, g\right) \sim\left(\left\{\psi_{n}\right\}, g\right)$.

Teorem 3.5. Chain homotopy relation in the category of fuzzy soft modules is a equivalence relation and is invariant according to composition. 
Proof. Primarily, we show that chain homotopy relation is an equivalence relation.

1) Let $(\varphi, g)=\left(\left\{\varphi_{n}\right\}, g\right):\left\{\left(F_{n}, A\right), \partial_{n}\right\} \rightarrow\left\{\left(G_{n}, B\right), \partial_{n}^{\prime}\right\}$ be an arbitrary morphism. If $D_{n}=0$ then $\varphi_{n}-\varphi_{n}=0$. That is $(\varphi, g) \sim(\varphi, g)$.

2) Let $(\varphi, g)$ with $(\psi, g)$ be a chain homotopy. That is,

$$
D_{n-1} \circ \partial_{n}+\partial_{n+1}^{\prime} \circ D_{n}=\varphi_{n}-\psi_{n}
$$

if $\bar{D}_{n=}-D_{n}$, in this case

$\bar{D}_{n-1} \partial_{n}+\partial_{n+1}^{\prime} \bar{D}_{n}=-D_{n-1} \partial_{n}-\partial_{n+1}^{\prime} D_{n}=-\left(, D_{n-1} \partial_{n}+\partial_{n+1}^{\prime} D_{n}\right)=-\left(\varphi_{n}-\psi_{n}\right)$

and then $(\varphi, g)$ whit $(\psi, g)$ is a chain homotopy.

3. Let $(\varphi, g)$ with $(\psi, g)$ and $(\psi, g)$ with $(\gamma, g)$ be a chain homotopy. We want to show that $(\varphi, g)$ with $(\gamma, g)$ is a chain homotopy. If $(\varphi, g)$ with $(\psi, g)$ is a chain homotopy

$\exists D_{n}=>D_{n-1} \partial_{n}+\partial_{n+1} D_{n}=\psi_{n}-\gamma_{n}$. If $(\psi, g)$ with $(\gamma, g)$ is a chain homotopy,

$\exists D_{n}^{\prime}=>D_{n-1}^{\prime} \partial_{n}+\partial_{n+1}^{\prime} D_{n}^{\prime}=\psi_{n}-\gamma_{n}$.

Let

define

$$
\begin{aligned}
& \text { the homomorphism } \\
& D_{n}^{\prime \prime} \partial_{n}+\partial_{n+1}^{\prime} D_{n}^{\prime \prime}= \\
& \left(D_{n-1}+D_{n-1}^{\prime}\right) \partial_{n}+\partial_{n+1}^{\prime}\left(D_{n}+D_{n}^{\prime}\right)= \\
& D_{n-1} \partial_{n}+D_{n-1}^{\prime} \partial_{n}+\partial_{n+1}^{\prime} D_{n}+\partial_{n+1}^{\prime} D_{n}^{\prime}= \\
& D_{n-1} \partial_{n}+\partial_{n+1}^{\prime} D_{n}+D_{n-1}^{\prime} \partial_{n}+\partial_{n+1}^{\prime} D_{n}^{\prime}= \\
& \left(\varphi_{n}-\psi_{n}\right)+\left(\psi_{n}-\gamma_{n}\right)= \\
& \left(\varphi_{n}-\gamma_{n}\right)
\end{aligned}
$$

Now, we show the invariance of composition.

$\left(\left\{\varphi_{0 n}\right\}, g\right) \sim\left(\left\{\psi_{0 n}\right\}, g\right):\left[\left\{\left(F_{n}, A\right), \partial_{n}\right\} \rightarrow\left\{\left(G_{n}, B\right), \partial_{n}^{\prime}\right\}\right]=>D_{n-1} \partial_{n}+\partial_{n+1}^{\prime} D_{n}=\varphi_{0 n}-\psi_{0 n}$

$\left(\left\{\varphi_{1 n}\right\}, h\right) \sim\left(\left\{\psi_{1 n}\right\}, h\right):\left[\left\{\left(G_{n}, B\right), \partial_{n}^{\prime}\right\} \rightarrow\left\{\left(P_{n}, C\right), \partial_{n}^{\prime \prime}\right\}\right]=>D_{n-1}^{\prime} \partial_{n}+\partial_{n+1}^{\prime \prime} D_{n}^{\prime}=\varphi_{1 n}-\psi_{1 n}$

For $\left(\left\{\varphi_{1 n}\right\}, h\right) \circ\left(\left\{\varphi_{0 n}\right\}, g\right),\left(\left\{\psi_{1 n}\right\}, h\right) \circ\left(\left\{\psi_{0 n}\right\}, g\right):\left[\left\{\left(F_{n}, A\right), \partial_{n}\right\} \rightarrow\left\{\left(P_{n}, C\right), \partial_{n}^{\prime \prime}\right\}\right]$ to be a chain homotopy we have to define a homomorphism in following the form

$$
\begin{gathered}
\left(D_{n}^{\prime \prime}, \omega\right):\left[\left\{\left(F_{n}, A\right), \partial_{n}\right\} \rightarrow\left\{\left(H_{n}, C\right), \partial_{n}^{\prime \prime}\right\}\right], D_{n}^{\prime \prime}=D_{n-1}^{\prime}\left(\varphi_{0 n-1}\right) \\
D_{n-1}^{\prime}\left(\varphi_{0 n-1}, g\right) \partial_{n}+\partial_{n+1}^{\prime \prime} D_{n}^{\prime}\left(\varphi_{0 n}, g\right)= \\
D_{n-1}^{\prime}\left(\partial_{n}^{\prime}\left(\varphi_{0 n}, g\right)\right)+\partial_{n+1}^{\prime \prime} D_{n}^{\prime}\left(\varphi_{0 n}, g\right)= \\
\left(D_{n-1}^{\prime} \partial^{\prime}+\partial_{n+1}^{\prime \prime} D_{n}^{\prime}\right)\left(\varphi_{0 n}, g\right)= \\
\left(\varphi_{1 n}, h\right)\left(\varphi_{0 n}, g\right)-\left(\psi_{1 n}, h\right)\left(\varphi_{0 n}, g\right)
\end{gathered}
$$


Now, we show that $\left(\left\{\psi_{1 n}\right\}, h\right) \circ\left(\left\{\varphi_{0 n}\right\}, g\right)$ with $\left(\left\{\psi_{1 n}\right\}, h\right) \circ\left(\left\{\psi_{0 n}\right\}, g\right)$ are chain homotopy. We are look at $\left(\psi_{1 n+1}, h\right) \circ D_{n}: F_{n}(a) \rightarrow P_{n+1}(h(g(a)))$.

$\left(\psi_{1 n}, h\right) D_{n-1} \partial_{n}+\partial_{n+1}^{\prime \prime}\left(\psi_{1 n+1}, h\right) D_{n}=\left(\psi_{1 n}, h\right) D_{n-1} \partial_{n}+\left(\psi_{1 n}, h\right) \partial_{n+1}^{\prime} D_{n}=\left(D_{n-1} \partial_{n}+\partial_{n+1}^{\prime} D_{n}\right)\left(\psi_{1 n}, h\right)=$ $\left(\left(\varphi_{0 n}, g\right)-\left(\psi_{0 n}, g\right)\right)\left(\psi_{1 n}, h\right)=\left(\varphi_{0 n}, g\right)\left(\psi_{1 n}, h\right)-\left(\psi_{0 n}, g\right)\left(\psi_{1 n}, h\right)$

Then $\left(\varphi_{0 n}, g\right)^{\circ}\left(\psi_{1 n}, h\right)$ with $\left(\psi_{1 n}, g\right)^{\circ}\left(\psi_{0 n}, h\right)$

is a chain homotopy. Hence, from the two equalities, $\left(\left\{\varphi_{1 n}\right\}, h\right)^{\circ}\left(\left\{\varphi_{0 n}\right\}, g\right)$ with $\left(\left\{\psi_{1 n}\right\}, h\right)^{\circ}\left(\left\{\psi_{0 n}\right\}, g\right)$ is a chain homotopy.

Let $(\mathcal{F}, A)=\left\{\left(F_{n}, A\right), \partial_{n}\right\}$ be a chain complex of fuzzy soft modules over $\left\{M_{n}\right\}$. We obtain the homology module $H_{n}\left(M_{n}, F_{n}(a)\right)=\left(k e r \partial_{n} / \operatorname{Im} \partial_{n+1}, \widetilde{F}_{n}(a)\right.$ for the chain complex $\left\{\left(M_{n}, F_{n}(a)\right), \partial_{n}:\left(M_{n}, F_{n}(a)\right) \rightarrow\left(M_{n-1}, F_{n-1}(a)\right)\right\}$ and $\forall a \in A$. Here $\widehat{F}_{n}(a)$ degree function of quotient fuzzy modules in $M_{n}$ module. If the exist an one-to-one and covered connection with every submodule of quotient module of $M_{n}$ and submodule of $M_{n}$ we can think the module $H_{n}\left(M_{n}, F_{n}(a)\right)$ as a fuzzy submodule of $M_{n}$. Thus $H_{n}\left(F_{n},-\right): A \rightarrow P\left(M_{n}\right)$ is a fuzzy soft module.

Definition 3.6. Fuzzy soft module $H_{n}(\mathcal{F}, A)$ is said to be $\mathrm{n}$-dimensional homology fuzzy soft module of chain complexes of fuzzy soft modules $\left\{\left(F_{n}, A\right), \partial_{n}\right\}$.

Now, we show that homology fuzzy soft module is functor. Let $\left(\varphi=\left(\left\{\varphi_{n}\right\}, g\right):\left\{\left(F_{n}, A\right), \partial_{n}\right\} \rightarrow\left\{\left(G_{n}, B\right), \partial_{n}^{\prime}\right\}\right.$ be morphisms of chain complexes of fuzzy soft modules. Since $\left\{\varphi_{n}:\left(M_{n}, F_{n}(a)\right) \rightarrow N_{n}, G_{n}(g(a))\right\}$ is morphism of chain complexes of fuzzy modules for all $a \in A$, mapping $\varphi_{n *}: H_{n}(F, a) \rightarrow H_{n}(G, g(a))$, defined by $\varphi_{n *}[x]=$ $\left[\varphi_{n}(x)\right]$ for all $[x] \in H_{n}(F, a)$, is a homomorphism of fuzzy modules, and the following diagram is commutative

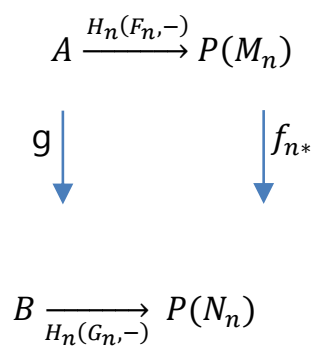

Then $\left.\left(\varphi_{n *}, g\right): H_{n}\left(F_{n},-\right), A\right) \rightarrow\left(H_{n}\left(G_{n},-\right), B\right)$ is homomorphism of fuzzy soft modules.

Theorem 3.7. The corresponding $(\mathcal{F}, A) \mapsto H_{n}(\mathcal{F}, A),\left(\left\{\varphi_{n}\right\}, g\right) \rightarrow\left(\varphi_{n}\right)_{*}$ is a covariant functor from category CCSM to the category FSM.

Theorem 3.8. Homology functor of chain complexes of fuzzy soft modules is invariant according to chain homotopy. That is, if $\left\{\varphi_{n}\right\} \sim\left\{\psi_{n}\right\}:\left\{\left(F_{n}, A\right), \partial_{n}\right\} \rightarrow\left\{\left(G_{n}, B\right), \partial_{n}^{\prime}\right\}$ then $\varphi_{n *}=\psi_{n *}=H_{n}(\mathcal{F}, A) \rightarrow H_{n}(G, A)$

Proof. Since $\left\{\varphi_{n}\right\}$ with $\left\{\psi_{n}\right\}$ is a chain homotopy for all $a \in A$, then

$\exists D_{n}:\left(F_{n}, A\right) \rightarrow\left(G_{n+1}, B\right)$ such that the equation

$D_{n-1} \partial_{n}+\partial_{n+1}^{\prime} D_{n}=\varphi_{n}-\psi_{n}$ is satisfied.

Now, we show that $\varphi_{n *}=\psi_{n *}: H_{n}(\mathcal{F}, A) \rightarrow H_{n}(G, A)$ is satisfied. For $\forall a \in A$ and

$\forall[z]=z+\operatorname{Im} \partial_{n+1} \in H_{n}\left(M_{n}, F_{n}(a)\right)$ we want to show that

$$
\varphi_{n *}\left(z+\operatorname{Im} \partial_{n+1}\right)=\psi_{n *}\left(z+\operatorname{Im} \partial_{n+1}\right) .
$$


That is $\varphi_{n *}\left(z+\operatorname{Im} \partial_{n+1}\right)=\varphi_{n}(z)+\operatorname{Im} \partial_{n+1}^{\prime}$

We show that

$$
\psi_{n *}\left(z+\operatorname{Im} \partial_{n+1}\right)=\psi_{n}(t)+\operatorname{Im} \partial_{n+1}^{\prime}
$$

Since $z \in k e r \partial_{n}$, and from the equation

$$
\begin{gathered}
D_{n-1} \partial_{n}(z)+\partial_{n+1}^{{ }_{n+1}} D_{n}(z)=\partial_{n+1}\left(D_{n}(z)\right)=\varphi_{n}(z)-\psi_{n}(z) \\
\Rightarrow a=\varphi_{n}(z)-\psi_{n}(z) \quad J b \in D_{n}(z) \quad \partial_{n+1}(b)=a \\
\Rightarrow a \epsilon J_{m} \partial^{\prime}{ }_{n+1} \\
\Rightarrow \varphi_{n}(z)-\psi_{n}(z) \epsilon J_{m} \partial_{n+1} \\
{\left[\varphi_{n}(z)\right]=\varphi_{n}(z)+J_{m} \partial_{n+1}} \\
{\left[\psi_{n}(z)\right]=\psi_{n}(z)+J_{m} \partial_{n+1}}
\end{gathered}
$$

Let $\left(F^{\prime}, A\right)$ is fuzzy soft module over $M^{\prime}$ and $\left(F^{\prime \prime}, A\right)$ is fuzzy soft module over $M^{\prime \prime}$

Definition 3.9 If for each $a \epsilon A$ sequence of fuzzy modules

$$
0 \rightarrow\left(M^{\prime}, F^{\prime}(a)\right) \stackrel{i_{a}}{\rightarrow}(M, F(a)) \stackrel{p_{a}}{\rightarrow}\left(M^{\prime \prime}, F^{\prime \prime}(a)\right) \rightarrow 0
$$

is short exact, then the sequence

$$
0 \rightarrow\left(F^{\prime}, A\right) \rightarrow(F, A) \rightarrow\left(F^{\prime \prime}, A\right) \rightarrow 0
$$

of fuzzy soft modules is said short exact sequence

Definition 3.10 If for each $a \in A$ sequence of fuzzy modules

$$
0 \rightarrow\left(M^{\prime}, F^{\prime}(a)\right) \rightarrow(M, F(a)) \rightarrow\left(M^{\prime \prime}, F^{\prime \prime}(a)\right) \rightarrow 0
$$

is splitting short exact sequence then

$$
0 \rightarrow\left(F^{\prime}, A\right) \rightarrow(F, A) \rightarrow\left(F^{\prime \prime}, A\right) \rightarrow 0
$$

is called splitting short exact sequence of fuzzy soft modules.

Teorem 3.11. If the sequence $\quad 0 \rightarrow\left(F_{n}^{\prime}, A\right) \rightarrow\left(F_{n}, A\right) \rightarrow\left(F_{n}{ }^{\prime \prime} A\right) \rightarrow 0$

is short exact sequence of fuzzy soft chain complexes, then the following sequence of fuzzy soft homology modules

$$
\ldots \leftarrow H_{n-1}\left(F_{n}, A\right) \stackrel{\partial_{*}}{\leftarrow} H_{n}\left(F_{n}{ }^{\prime}, A\right) \leftarrow H_{n}\left(F_{n}, A\right) \leftarrow H_{n}\left(F_{n}{ }^{\prime}, A\right) \ldots
$$

is exact.

Proof: Firstly, we prove that the sequence of homology modules of chain complexes is exact and homomorphism is fuzzy homomorphism. Since the homomorphism 
$\partial_{* n}: H_{n}\left(M_{n}{ }^{\prime \prime}, F_{n}^{\prime \prime}(a)\right) \rightarrow H_{n-1}\left(M_{n-1}^{\prime}, F_{n-1}^{\prime}(a)\right) \quad$ is not homomorphism of fuzzy modules. Sequence of fuzzy homology modules (3) is not generally exact. Since fuzzy short exact sequence (2) is fuzzy splitting, there exist fuzzy homomorphisms

$$
J_{n}:\left(\left(F_{n}^{*}, A\right) \rightarrow\left(F_{n}^{\prime}, A\right), q_{n}:\left(F_{n}^{\prime \prime}, A\right) \rightarrow\left(F_{n}, A\right), \forall n \in Z\right.
$$

such as

$$
J_{n} \circ i_{n}=\left(1_{\left(F_{n}, A\right)}\right), P_{n} \circ q_{n}=1_{\left(F_{n}^{\prime \prime}, A\right)}, i_{n} \circ j_{n}+, q_{n} \circ p_{n}=1_{\left(F_{n}, A\right)}
$$

then

$$
\bar{d}_{n}=j_{n-1} \circ \partial_{n} \circ p_{n}:\left(F_{n}{ }^{\prime}, A\right) \rightarrow\left(F_{n-1}^{\prime}, A\right) \forall n \in Z
$$

is a fuzzy soft homomorphism of fuzzy soft modules, and the family

$$
\bar{d}_{n}=\left\{d_{n}:\left(F_{n}{ }^{\prime \prime}, A\right) \rightarrow\left(F_{n}{ }^{\prime}, A\right)\right\}
$$

is fuzzy soft homomorphism of fuzzy soft chain complexes having a degree of " -1 ". Indeed, for the homomorphisms $\bar{d}_{n}=\left\{d_{n}:\left(F_{n}{ }^{\prime}, A\right) \rightarrow\left(F_{n}{ }^{\prime}, A\right)\right\}$, the following

$$
\begin{gathered}
i_{n-2}\left(\partial_{n-1}^{\prime} d_{n}\right)=\left(i_{n-2} \partial_{n-1}^{\prime}\right) J_{n-1} \partial_{n} q_{n}= \\
\partial_{n-1}\left(i_{n-1} J_{n-1}\right) \partial_{n} q_{n}=\partial_{n-1}\left(i_{n-1}-q_{n-1} p_{n-1}\right) \partial_{n} q_{n}= \\
\partial_{n-1} \partial_{n} q_{n}-\partial_{n-1} q_{n-1} P_{n-1} \partial_{n} q_{n}=-\partial_{n-1} q_{n-1} p_{n-1} \partial_{n} q_{n}= \\
-\partial_{n-1} q_{n-1}\left(p_{n-1} \partial_{n}\right) q_{n}=-\partial_{n-1} q_{n-1} \partial_{n}{ }^{\prime} P_{n} q_{n}= \\
-\partial_{n-1} q_{n-1} \partial_{n}{ }_{n} 1_{c^{\prime \prime} n}=-\partial_{n-1} q_{n-1} \partial_{n}{ }_{n}= \\
-\left(i_{n-2} J_{n-2}+q_{n-2} p_{n-2}\right) \partial_{n-1} q_{n-1} \partial_{n}{ }^{\prime \prime}= \\
-i_{n-2}\left(J_{n-2} \partial_{n-1} q_{n-1}\right) \partial_{n}-q_{n-2}\left(p_{n-2} \partial_{n-1}\right) q_{n-1} \partial_{n}{ }^{\prime}= \\
-i_{n-2}\left(d_{n-1} \partial_{n}\right)-q_{n-2} \partial_{n-1}\left(P_{n-1} q_{n-1}\right) \partial_{n}= \\
-i_{n}\left(d_{n-1} \partial_{n}\right)
\end{gathered}
$$

imply that $\partial_{n-1} d_{n}=d_{n-1} \partial_{n}^{\prime \prime}$ is satisfied since $i_{n-2}$ is a monomorphism, that is, the family $\left\{d_{n}\right\}$ is a morphism of chain complexes [2] since $d_{n}:\left(F_{n}{ }^{\prime \prime}, A\right) \rightarrow\left(F_{n-1}{ }^{\prime}, A\right)$ is a fuzzy soft homomorphism, $\tilde{d}_{n}:\left(F_{n}{ }^{\prime}, A\right) \rightarrow\left(F_{n}{ }^{\prime}, A\right)$ is fuzzy homomorphism of fuzzy chain complexes

For each $[z] \epsilon H_{n}\left(c^{\prime \prime}\right)$

$\left.\partial_{* n}(z)=\left[i_{n-1}{ }^{-1} \circ \partial \circ j_{n}{ }^{-1}(z)\right]=\left[J_{n-1} \circ\right] \partial_{n} \circ q_{n}(z)\right]=\left[d_{n}(z)\right]=d_{n *}(z)$,

These $\bar{\partial}_{n}: H_{n}\left(M_{n}{ }^{\prime}, F^{\prime}(a)\right) \rightarrow H_{n-1}\left(M_{n}{ }^{\prime} F^{\prime}(a)\right)$ is a fuzzy homomorphism of fuzzy modules. Therefore, the sequence (2) is exact.

Proposition 3.12. For each split fuzzy soft short exact sequence of fuzzy soft modules 


$$
0 \rightarrow(F, A) \stackrel{\alpha}{\rightarrow}(P, A) \stackrel{\beta}{\rightarrow}(Q, A) \rightarrow 0
$$

over $M$, and each fuzzy soft module $(G, B)$ over $N$ the sequence

$$
0 \rightarrow(F, A) \otimes(G, B) \rightarrow(P, A) \otimes(G, B) \rightarrow(Q, A) \otimes(G, B) \rightarrow 0
$$

is fuzzy split short exact sequence.

Proof: In order to prove proposition, it is enough to demonstrate that the fuzzy soft homomorphism

$\bar{\alpha} \otimes \overline{1} \quad$ has a left inverse. The fuzzy soft homomorphism $\bar{\alpha}$ has a left inverse $\bar{\alpha}$. Hence the fuzzy homomorphism $\bar{\alpha} \otimes \widetilde{1}$ is the left inverse of the fuzzy soft homomorphism $\bar{\alpha} \otimes \overline{1}$. Since tensor product in category of fuzzy soft modules is a functor for each fuzzy chain complex

$\left(\mathcal{F}_{n}, A\right)=\left\{\left(\mathcal{F}_{n}, A\right), \tilde{\partial}_{n}\right\}$ and each fuzzy soft $(G, B)$ over module $N$ the family

$$
\left(F_{n}, A\right) \otimes(G, B) \rightarrow\left\{\left(F_{n}, A\right) \otimes(G, B), \tilde{\partial}_{n} \otimes \overline{1}_{(G, B)}\right\}
$$

Is a fuzzy soft chain complexes of fuzzy soft modules?

Definition 3.13. Fuzzy soft homology module $H_{n}((\mathcal{F}, A) \otimes(G, B)$ is called homology module with coefficient $(G, B)$ of fuzzy chain complex and is represented by

$H_{n}((\mathcal{F}, A) ;(G, B))$.

From proposition 2.11, for each split short exact sequence of fuzzy chain complexs

$$
0 \rightarrow\left(\mathcal{F}^{\prime}, A\right) \rightarrow(\mathcal{F}, A) \rightarrow\left(\mathcal{F}^{\prime \prime}, A\right) \rightarrow 0
$$

and each fuzzy soft module $(G, B)$, the sequence

$$
0 \rightarrow\left(F^{\prime}, A\right) \otimes(G, B) \rightarrow(F, A) \otimes(G, B) \rightarrow\left(F_{n}{ }^{\prime \prime}, A\right) \otimes(G, B) \rightarrow 0
$$

is a split fuzzy soft short exact sequence. Then by using Theorem 2.9, we can easily prove the following theorem.

Teorem 3.14. For each split short exact sequence of fuzzy soft chain complex

$$
0 \rightarrow\left(\mathcal{F}^{\prime}, A\right) \rightarrow(\mathcal{F}, A) \rightarrow\left(\mathcal{F}^{\prime \prime}, A\right) \rightarrow 0
$$

and each fuzzy module $(G, B)$, the sequence of fuzzy soft homology modules.

$$
\ldots \leftarrow H_{n-1}\left(\left(\mathcal{F}^{\prime}, A\right) ;(G, B)\right) \leftarrow H_{n}\left(\left(F \quad{ }^{\prime}, A\right) ;(G, B)\right) \leftarrow H_{n}((\mathcal{F}, A) ;(G, B)) \leftarrow \leftarrow H_{n}\left(\left(F_{n}{ }^{\prime}, C^{\prime}\right) ;(G, B)\right) \leftarrow \cdots
$$

is exact and functorial.

Let $(\mathcal{F}, A)=\left\{\left(F_{n}, A\right), \tilde{\partial}_{n}\right\}$ be fuzzy soft chain complexes and $(G, B)$ be fuzzy soft module. We can show that the homomorphism of fuzzy soft module

$\varphi_{n}: H_{n}(\mathcal{F}, A) \otimes(G, B) \rightarrow H_{n}((\mathcal{F}, A) ;(G, B))$, for each $a \in A, b \in B \quad \varphi_{n}([z], g)=[z \otimes g]$

is a fuzzy homomorphism.

For each $\quad[z] \otimes g \epsilon H_{n}(C) \otimes G$ 


$$
\begin{gathered}
\left(\left(\tilde{F}_{n}(a)\right) \otimes(G(b))([z] \otimes g)=\vee\left(\left(\bar{F}_{n}(a)\right) \times(G(b)) \vee([z], g)==\vee_{\left(z^{\prime}, \mathrm{g}\right) \in[\mathrm{z}] \otimes g}\left(\left(F_{n}(a)\right) z^{\wedge} \wedge(G, B) g\right)\right.\right. \\
\left(F_{n}(a)\right) \otimes(G(b))\left(\varphi_{n}[z] \otimes g\right)=\left(\left(F_{n}(a)\right) \otimes(G(b))=\right. \\
\vee_{\left(z^{\prime}, g^{\prime}\right) \in[z \otimes g]}\left(\left(F_{n}(a)\right) z^{\prime \prime} \wedge(G(b)) g^{\prime}\right)
\end{gathered}
$$

It is a $[z] \otimes g \subset[z \otimes g]$, then

$$
\left.\left(\left(F_{n}(a)\right) \otimes(G(b))\right)([z] \otimes g) \leq\left(F_{n}(a)\right) \otimes(G(b))\right)\left(\left(\varphi_{n}[z] \otimes g\right)\right)
$$

and $\bar{\varphi}_{n}$ is a fuzzy homomorphism

Let $R$ be a principial ideal domain and $R$ be a commutative ring $[15,17]$

Teorem 3.15. If $(\mathcal{F}, A)$ is a free fuzzy soft chain complex and $(G, B)$ is a fuzzy soft module then there is a functorial fuzzy short exact sequence

$$
0 \rightarrow H_{n}\left(( \mathcal { F } , A ) \otimes ( G , b ) \stackrel { \overline { \varphi } _ { n } } { \rightarrow } H _ { n } \left((\mathcal{F}, A) ;(G, B) \rightarrow F S-\operatorname{Tor}\left(H_{n-1}((\mathcal{F}, A),(G, B)) \rightarrow 0\right.\right.\right.
$$

and this sequence a split.

Proof. Let for $\forall a \in A$

$$
\begin{gathered}
Z\left(M_{n}, F_{n}(a)\right)=\left\{\operatorname{Ker} \bar{\partial}_{n} \subset\left(M_{n}, F_{n}(a)\right)\right. \\
B\left(M_{n+1}, F_{n+1}(a)=\left\{\operatorname{Im} \bar{\partial}_{n} \subset\left(M_{n}, F_{n}(a)\right)\right.\right.
\end{gathered}
$$

be the subcomplex of $(\mathcal{F}, A)$. The operator " 0 " is boundary operator of these subcomplexes. Since $R$ is a principial ideal domain, both $Z\left(M_{n}, F_{n}(a)\right)$ and $B\left(M_{n+1}, F_{n+1}(a)\right.$ are free fuzzy chain complexes and there is a fuzzy short exact sequence.

$$
0 \rightarrow Z\left(M_{n}, F_{n}(a)\right) \stackrel{\alpha}{\rightarrow}\left(M_{n}, F_{n}(a)\right) \stackrel{\beta}{\rightarrow} B\left(M_{n+1}, F_{n+1}(a)\right) \rightarrow 0
$$

where the fuzzy homomorphisms

$\widetilde{\alpha}_{n}:\left(\left(F_{n}, Z(c)\right) \rightarrow\left(F_{n} C_{n}\right)\right.$

are induced from the homomorphisms

$$
\tilde{\beta}_{n}:\left(\left(F_{n}, A\right) \rightarrow\left(F_{n}, B_{(n-1)(\varepsilon n)}\right)\right.
$$

$\alpha_{n}(z)=z, \quad \beta_{n}(c)=\partial_{n}(c)$ for each $n \in Z$. Since $\left\{B\left(M_{n+1}, F_{n+1}(a)\right)\right\}$ is a free fuzzy soft chain complex, the short exact sequence (4) is split. Hence from theorem 3.11, the following fuzzy soft exact sequence is obtain

$$
\begin{gathered}
\ldots \rightarrow H_{n}\left(Z\left(M_{n}, F_{n}(a)\right) ;(G, B)\right) \rightarrow H_{n}\left(\left(M_{n}, F_{n}(a)\right) ;(G, B)\right) \rightarrow H_{n}\left(B\left(\left(M_{n+1}, F_{n+1}(a)\right) ;(G, B)\right)\right. \\
\rightarrow H_{n-1}\left(Z\left(M_{n-1}, F_{n-1}(a)\right) ;(G, B)\right) \rightarrow \cdots
\end{gathered}
$$

Since the fuzzy soft chain complex $\left(B\left(M_{n+1}, F_{n+1}(a)\right.\right.$ have trivial boundary operators, the boundary operators of fuzzy soft chain complexes $\left(Z\left(M_{n}, F_{n}(a)\right) \otimes(G, B)\right.$ and $\left(B\left(M_{n+1}, F_{n+1}(a) \otimes(G, B)\right.\right.$ are trivial too. Therefore, we have

$$
\begin{array}{r}
H_{n}\left(Z\left(M_{n}, F_{n}(a)\right) ;(G, B)\right) \rightarrow H_{n}\left(\left(M_{n}, F_{n}(a)\right) \otimes(G, B)\right. \\
H_{n}\left(B\left(\left(M_{n}, F_{n}(a)\right) ;(G, B)\right) \rightarrow H_{n}\left(B\left(M_{n-1}, F_{n-1}(a)\right) \otimes(G, B)\right)\right.
\end{array}
$$

hence the fuzzy soft exact sequence (5) turns into the fuzzy soft exact sequence 


$$
\begin{aligned}
\ldots \rightarrow\left(B \left(M_{n+1,} F_{n+1}\right.\right. & (a)) \otimes(G, B) \stackrel{\tilde{J}_{n} \otimes 1_{(G, B)}}{\longrightarrow}\left(Z\left(M_{n}, F_{n}(a)\right) \otimes(G, B)\right. \\
& \rightarrow H_{n}\left(M_{n},\left(F_{n}(a)\right) ;(G, B)\right) \\
& \rightarrow\left(B ( M _ { n , } F _ { n } ( a ) ) \otimes ( G , B ) \stackrel { j _ { n - 1 } \otimes 1 _ { ( G , B ) } } { \longrightarrow } Z \left(M_{n-1}, F_{n-1}(a) \otimes(G, B) \rightarrow \cdots\right.\right.
\end{aligned}
$$

Where $\tilde{J}_{n}:\left(\left(B\left(M_{n+1}, F_{n+1}(a)\right) \rightarrow Z\left(M_{n}, F_{n}(a)\right)\right.\right.$ is fuzzy soft embedding homomorphism. From sequence (6) we obtain the following fuzzy soft short exact sequence

$$
0 \rightarrow \operatorname{coker}\left(\tilde { J } _ { n } \otimes 1 _ { ( G , B ) } \rightarrow H _ { n } ( M _ { n } , ( F _ { n } ( a ) ) ; ( G , B ) ) \rightarrow \operatorname { k e r } \left(j_{n-1} \otimes 1_{(G, B)} \rightarrow 0\right.\right.
$$

Now, let us consider fuzzy soft short exact sequence of fuzzy modules

$$
0 \rightarrow B\left(M_{n+1}, F_{n+1}(a)\right) \rightarrow\left(M_{n}, F_{n}(a)\right) \rightarrow H_{n}\left(M_{n}, F_{n}(a)\right) \rightarrow 0
$$

Since $Z\left(M_{n}, F_{n}(a)\right)$ is a fuzzy soft free module, there is the following fuzzy soft exact sequence

$$
\begin{gathered}
\left.0 \rightarrow F S-\operatorname{TorH}_{n}\left(M_{n}, F_{n}(a)\right) ;(G, B)\right) \rightarrow B\left(\left(M_{n+1}, F_{n+1}(a)\right) \otimes(G, B)\right) \\
\rightarrow 0
\end{gathered} \rightarrow H_{n}\left(\left(M_{n}, F_{n}(a)\right) \otimes(G, B)\right)
$$

From the sequence (8)

$$
\begin{gathered}
\operatorname{coker}\left(\tilde{J}_{n} \otimes 1_{(G, B)}\right)=H_{n}\left(M_{n},\left(F_{n}(a)\right)\right) \otimes(G, B)=H_{n}\left(M_{n}, F_{n}(a)\right) \otimes(G, B) \\
\operatorname{ker}\left(\tilde{J}_{n} \otimes 1_{(G, B)}\right)=F S-\operatorname{Tor}\left(M_{n}, F_{n}(a)\right) ;(G, B)
\end{gathered}
$$

Substituting these with (7), the fuzzy soft short exact sequence

$$
0 \rightarrow H_{n}\left(( \mathcal { F } , A ) \otimes ( G , b ) \stackrel { \overline { \varphi } _ { n } } { \rightarrow } H _ { n } \left((\mathcal{F}, A) ;(G, B) \rightarrow F S-\operatorname{Tor}\left(H_{n-1}((\mathcal{F}, A),(G, B)) \rightarrow 0\right.\right.\right.
$$

Can be obtained.

If $\tilde{\tau}:(\mathcal{F}, A) \rightarrow\left(\mathcal{F}^{\prime}, A\right)$ is a fuzzy soft morphism of fuzzy soft chain complexes, the following commutative diagram.

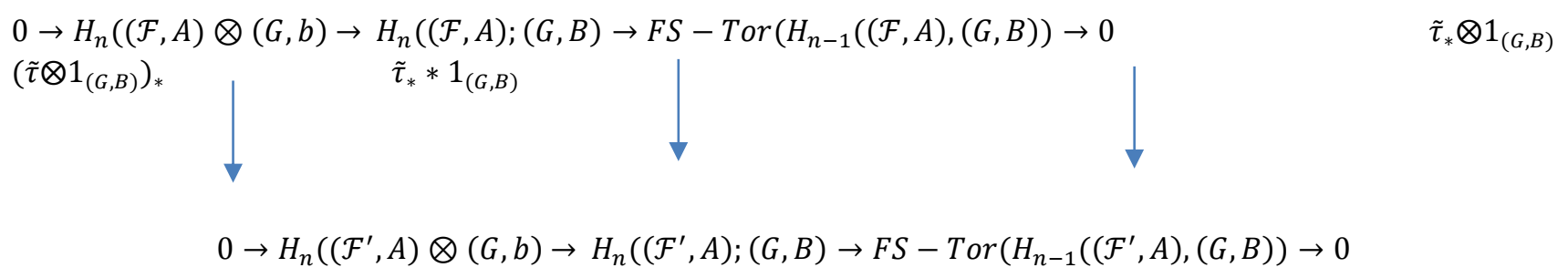

is obtained, since the fact that the operations $H_{n}, \otimes, F S-$ Tor are functors. There fore it is proved that fuzzy soft short exact sequence in the theorem in functorial.

\section{References}

1. U. Acar, F. Koyuncu, and B. Tanay, Soft sets and soft rings, Comput. Math. Appl. 59 (2010) 3458-3463.

2. H. Aktaş and N. Çagman, Soft sets and soft group, Inform. Sci. 177 (2007) 2726-2735.

3. S.A. Bayramov, Fuzzy and fuzzy soft structures in algebras, Lambert Academic Publishing, 2012. 
4. F. Feng, Y.B. Jun and X. Zhao, Soft semirings, Comput. Math. Appl. 56 (2008) 2621-2628.

5. C. Gunduz (Aras) and S. Bayramov, Fuzzy soft modules, Int. Math. Forum 6(11) (2011) 517-527.

6. C. Gunduz (Aras), S.A. Bayramov, Intuitionistic fuzzy soft modules, Computers and Mathematics with Application, 62 (2011) 2480-2486.

7. L. Jin-liang, Y. Rui-xia and Y. Bing-xue, Fuzzy soft sets and fuzzy soft groups, Chinese Control and Decision Conference (2008) 2626-2629.

8. P.K. Maji, A.R. Roy and R. Bismas, Fuzzy soft set, The Journal of Fuzzy Mathematics 9 (2001) 589-602.

9. P.K. Maji, A.R. Roy and R. Bismas, An application of soft sets in a decision making problem, Comput. Math. Appl. 44 (2002) 1077-1083.

10. D. Molodtsov, Soft set theory-first results, Comput. Math. Appl. 37 (1999) 19-31.

11. A. Rosenfeld, Fuzzy groups, J. Math. Anal. Appl. 35 (1971) 512-517.

12. A.R. Roy and P.K. Maji, A fuzzy soft set theoretic approach to decision making problems, J. Comput. Appl. Math. 203 (2007) 412-418.

13. Sadi Bayramov, Cigdem Gunduz (Aras) . The Universal Coefficient Theorem for Fuzzy Homology Modules, Fuzzy sets, Rough Sets and multivalued oper. and Appl. (2011), No1, 41-50

14. R. Ameri, M.M. Zahedi, "Fuzzy Chain Complex and Fuzzy Homotopy", Fuzzy Sets and Systems, 112 (2000), $287-297$.

15. M.M. Zahedi, R. Ameri "Fuzzy Exact Sequence in Category of Fuzzy Modules", J. Fuzzy Math. ,3(1) (1995), $181-190$

16. Taha Yasin Ozturk, Sadi Bayramov. Category of chain complexes of soft Modules International mathematical forum 7. 2012 , No 40, 981-992

17. E. Spanier, "Algebraic Topology" McGRANE, New York, 1995.

18. S.E. Abdullayev, Sadi Bayramov, Inverse system in the category of intuitionistic fuzzy soft modules. Journal of Advances in Mathematics 18.03.2018, 7486-7502

19. C. Gunduz Aras, B.Davvaz, The Universal coefficient theorem of intuitionistic fuzzy modules, Utilitas Mathematica 81, pp 131-156 (2010)

20. Qiu-Mei Sun, Zi-Liong Zhang and Jing Liu, Soft sets and soft modules, Lecture Notes in Comput. Sci. 5009 (2008) 403-409.

21. S.R. Lopez-Permouth, D.S. Malik, On Categories of Fuzzy Modules, Information Sciences,72, (1993), 65-82

22. L.A.Zadeh, Fuzzy sets, Inform.\&Control, 8(1965),338-353. 